\title{
NECROLÓGIO
}

\section{Amilcar Vianna Martins (1907 - 1990)}

Aos 82 anos de idade faleceu em Belo Horizonte - Professor Amilcar Vianna Martins, um dos mais distinguidos parasitologistas brasileiros. Médico, formado pela Faculdade de Medicina da Universidade Federal de Minas Gerais (UFMG). Na UFMG foi Assistente, Livre-Docente em Parasitologia, Professor Catedrático de Zoologia, membro do Conselho Universitário e Professor Emérito. $\mathrm{Na}$ Venezuela foi Professor Titular Visitante na Universidad Central ena Universidad Centro-Occidental. Dirigiu o Serviço de Endemias Rurais do Estado de Minas Gerais (1947 - 1949), o Instituto Nacional de Endemias Rurais (1956 1958), o Instituto Oswaldo Cruz, Rio de Janeiro (1958 - 1960) e o Departamento Nacional de Endemias Rurais (1960 - 1961). Membro do Conselho Nacional de Saúde (1958 - 1961). Perito em Doenças Parasitárias da Organização Mundial de Saúde (1952 - 1960). Consultor da OMS sobre Sistemática e Biologia dos flebotomíneos. Presidente da Sociedade Brasileira de Parastitologia (1967) e da Sociedade de Biologia de Minas Gerais (1971). Membro Titular da Academia Brasileira de Ciências (1976) e Emérito da Academia Mineira de Medicina. Recebeu o prêmio "Costa Lima da Academia Brasileira de Medicina (1974) e as Medalhas de Grande Oficial da Ordem do Mérito Médico do Brasil (1957), do Jubileu de Prata da SBPC (1973) por Relevantes Serviços Prestados à Ciência Brasileira", as Medalhas Culturais Carlos Chagas, Pirajá da Silva e Gaspar Viana, por seus trabalhos relativos à doença de Chagas, esquistossomose e leishmaniose.

Serviu na Itália durante a $2^{\mathrm{a}}$ Grande Guerra e foi condecorado com a Medalha de Guerra e de Campanha da Força Expedicionária Brasileira.

O Professor Amilcar Vianna Martins era dotado de rara inteligência, espírito de observação, juízo crítico e capacidade de síntese. Modesto, afável mas firme na defesa do que lhe parecia justo, mesmo à custa de prejuízos pessoais. Nas pesquisas sobre a doença de Chagas contaminouse pelo Trypanosoma cruzi. Uma saudação em praça pública foi o ponto de partida para que dirigentes intolerantes o privassem de seus direitos políticos e o aposentassem compulsoriamente.
Sua atuação científica o coloca entre os maiores vultos da Medicina Tropical do Brasil.

A Revista publica a seguir a lista de seus trabalhos científicos:

1. Bastos AT, Martins AV. Sobre a distribuição dos grupos sanguíneos entre os Índios botocudos do Rio Doce. Brasil Médico 49:108-109, 1935.

2. Martins AV. Sobre a pesquisa dos ovos de Schistosoma mansoni pelo método da sedimentaçãoconcentração. Brasil Médico 51:309-321, 1937.

3. Dias E, Martins AV, Ribeiro DJ. Aspectos do tifo exantemático em Minas Gerais. Brasil Médico 51, 1937.

4. Dias E, Martins AV, Ribeiro DJ. Tifo exantemático no oeste de Minas Gerais. Brasil Médico 51, 1937.

5. Dias E, Martins AV. Preparação da vacina Spencer e Parker contra a febre maculosa das Montanhas Rochosas. O Hospital 13:55-70, 1938.

6. Martins AV, Versiani V. Schistosoma mansoni no norte de Minas Gerais. Brasil Médico 52:812-817, 1938.

7. Martins AV, Versiani V. Schistosoma mansoni em Belo Horizonte. Brasil Médico 52:471-472, 1938.

8. Martins AV. Contribuição ao estudo do Gênero Australorbis Pilsbry, 1934. Tese de Livre Docência de Parasitologia, Universidade Federal de Minas Gerais, Belo Horizonte, 1938

9. Dias E, Martins AV. Spotted fever in Brazil. The American Journal of Tropical Medicine and Hygiene 19:103-108, 1939

10. Martins AV, Versiani V. Plano de combate à schistosomose mansoni em Belo Horizonte. O Hospital 15:563-570, 1939.

11. Martins AV, Versiani V, Tupynambá AA. Estudos sobre a tripanosomíase americana em Minas Gerais. Memórias do Instituto Oswaldo Cruz 35:285-301, 1940.

12. Lent $\mathrm{H}$, Martins AV. Estudos sobre os triatomíneos do Estado de Minas Gerais, com a descrição de uma espécic nova. Revista de Entomologia 11:877. 886, 1940.

13. Martins AV. Os Tabanídeos do Estado de Minas Gerais. Tese de concurso para Professor Catedrático de Zoologia e Parasitologia, Universidade Federal de Minas Gerais, Belo Horizonte, 1940.

14. Martins AV, Tupynambá AA. Sobre dois casos agudos de moléstia de Chagas observados em Minas Gerais. Brasil Médico 54:839-841, 1940.

15. Martins AV. Infecção experimental do Triatoma arthurneivai Lent \& Martins, 1940, pelo Schizotrypanun cruzi. Brasil Médico 55:131, 1941.

16. Martins AV. Sobrea ocorrência da medusa de água doce Craspedacusta sowerbyi Lankester, 1880, 
Necrológio. Amilcar Vianna Martins (1907-1990). Revista da Sociedade Brasileira de Medicina Tropical 24:191195, jul-set, 1991

em Minas Gerais. Revista Brasileira de Biologia 1:227-230, 1941 .

17. Martins AV, Versiani V, Tupynambá AA. Sobre 25 casos agudos de moléstia de Chagas observados em Minas Gerais. Memórias do Instituto Ezequiel Dias 3/4:50-70, 1942.

18. Martins AV. Nova espécie do gênero Spiniger Burmeister, 1835 (Hemiptera, reduviidae). Revista Brasileira de Biologia 2:233-234, 1942.

19. Martins AV, Macedo E. Nota sobre a moléstia de Chagas na Bolívia. Brasil Médico 56:592-593, 1942.

20. Martins AV. Anaveneno escorpiônico. Brasil Médico 57:248-251, 1943.

21. Martins AV. Estudos sobre a filariose bancrofti na região amazônica. Revista Brasileira de Medicina Militar 33:223-232, 1944.

22. Martins AV. Sobre o uso do anaveneno na produção do soro antiescorpiônico. Arquivos do Instituto Químico-Biológico 1:7-26, 1945.

23. Martins AV, Versiani V, Tupynambá AA. Estudos sobre a moléstia de Chagas no Estado de Minas Gerais. I. Estudo epidemiológico de um foco da moléstia no Município de Jaboticatubas. Arquivos do Instituto Químico-Biológico 1:51-61, 1945.

24. Martins AV, Versiani V, Tupynambá AA. Estudos sobre a moléstia de Chagas no Estado de Minas Gerais. II. Sobre 156 xenodiagnósticos feitos em Belo Horizonte. Arquivos do Instituto QuímicoBiológico 1:63-70, 1945.

25. Versiani V, Martins AV, Pena Sobrinho $O$. Esquistossomose mansônica no Estado de Minas Gerais. I. Município de Belo Horizonte. Arquivos do Instituto Químico-Biológico 1:71-92, 1945.

26. Martins AV, Versiani V. Novo foco de malária quartã em Minas Gerais. Brasil Médico 60, 1946.

27. Martins AV. Terapêutica das esquistossomoses. In: Cardini C (ed) Tratado de Terapeutica Clínica, Editorial El Ateneo, Buenos Aires p. 182-196, 1946.

28. Martins AV, Freitas MG. Estudos sobre a habronemose equina. Boletim da Sociedade Brasileira de Medicina Veterinária 17:9-14, 1948.

29. Martins AV. Diagnóstico de laboratório da esquistossomose mansoni. Tese de concurso para Professor Catedrático de Parasitologia, Universidade Federal de Minas Gerais, Belo Horizonte, 1949.

30. Martins AV, Versiani V, Peres JN. Distribuição dos triatomíneos e seus índices de infecção pelo Schizotrypanum cruzi no Estado de Minas Gerais. Arquivos de Saúde Pública de Minas Gerais 2:6379, 1951.

31. Martins AV. Plano de tratamento em massa. In: Esquistossomose mansoni no Brasil. Debates promovidos pela Sociedade de Gastroenterologia e Nutrição de São Paulo p.143-150, 1952.

32. Martins AV, Filizzola Filho B. Considerações em torno da biópsia retal na esquistossomose. $l n$ : Temas Atuais de Proctologia, Rio de Janeiro p.185-194, 1952.

33. Martins AV, Martins G, Falcão AL. Distribuição dos planorbídeos e seus índices de infecção pelo Schistosoma mansoni no Município de Belo Horizonte. Trabalho apresentado ao XI Congresso Brasileiro de Higiene, Curitiba, Paraná, 1953.

34. Martins AV, Martins G, Brito RS. Os marsupiais como reservatórios da esquistossomose mansoni. Revista Brasileira de Medicina 2:165-166, 1954.

35. Friedheim EAH, Silva JR, Martins AV. Treatment of schistosomiasis mansoni with antimony-a dimercato-potassium succinide. The American Journal of Tropical Medicine and Hygiene 3:714:727, 1954.

36. Martins AV, Martins G, Brito RS. Reservatórios silvestres do Schistosoma mansoni no Estado de Minas Gerais. Revista Brasileira de Malariologia e Doenças Tropicais 7:259-265, 1955.

37. Martins AV. Tratamento da forma aguda da esquistossomose mansoni. Segunda Jornada Brasileira sobre Esquistossomose, Belo Horizonte, 1955.

38. Martins AV e col. Calazar autóctone em Minas Gerais. Revista Brasileira de Malariologia e Doenças Tropicais 8:555-563, 1956.

39. Martins AV e col. Observações preliminares sobre um foco de leishmaniose tegumentar em Minas Gerais. Revista Brasileira de Malariologia e Doenças Tropicais 8:577-581, 1956.

40. Barretto MP, Martins AV, Pellegrino J. Notas sobre flebotomíneos do nordeste do Estado de Minas Gerais, com as descrições de duas espécies novas (Diptera, Psychodidae). Folia Clinica et Biologica, São Paulo 26:47-56, 1956.

41. Martins AV, Falcão AL, Silva JE. Estudos sobre os flebótomos do Estado de Minas Gerais. I. Phlebolomus renei n.sp. (Diptera, Psychodidae). Revista Brasileira de Malariologia e Doenças Tropicais 9:321-325, 1957.

42. Barretto MP, Martins AV, Pellegrino J. Notas sobre os flebotomíneos do Nordeste do Estado de Minas Gerais, com as descrições de duas espécies novas (Diptera, Psychodidae). Revista Brasileira de Malariologia e Doenças Tropicais 10:183-193 (republicação), 1958.

43. Martins AV. Non-human vertebrate hosts of Schistosoma haematobium and Schistosoma mansoni. Bulletin of the the World Healt Organization, Genéve 18:931-944, 1958.

44. Martins AV, Souza MA, Silva JE. Estudos sobre 
Necrológio.Amilcar Vianna Martins (1907-1990). Revista da Sociedade Brasileira de Medicina Tropical 24:191195, jul-set, 1991

os flebótomos do Estado de Minas Gerais. II. Descrição do macho e redescrição da fêmea da Lutzomyia cavernicola (Costa Lima, 1932) (Diptera, Psychodidae). Revista Brasileira de Biologia 21:225-232, 1961.

45. Martins AV, Falcão AL, Silva JE. Estudos sobre os flebótomos do Estado de Minas Gerais. III. Descrição da Lutzomyia appendiculata sp.n. (Diptera, Psychodidae). Revista Brasileira de Biologia 21:297-300, 1961.

46. Martins AV, Godoy TL, Silva JE. Notas sobre os flebótomos dos Estados do Paraná e Santa Catarina, com redescrição da Lutzomyia gaminarai (Cordero, Vogelsang \& Cossio, 1928) (Diptera, Psychodidae). Revista Brasileira de Biologia 21:309-316, 1961.

47. Martins AV, Souza MA, Falcão AL. Estudos sobre os flebótomos do Estado de Minas Gerais. IV. Descrição de duas novas espécies do subgênero Lutzomyia França, 1924 (Diptera, Psychodidae). Revista Brasileira de Biologia 22:39-46, 1962.

48. Martins AV, Godoy TL, Silva JE. Nota sobre os flebótomos de Petrópolis, Estado do Rio de Janeiro, com a descrição de uma nova espécie (Diptera, Psychodidae). Revista Brasileira de Biologia 22:55-60, 1962.

49. Martins AV, Godoy TL, Silva JE. Uma nova espécie de flebótomo dos Estados do Rio de Janeiro e Espírito Santo-Luizomyiagasparviannai n.sp. (Diptera, Psychodidae). Revista do Instituto de Medicina de São Paulo. 4:85-90, 1962.

50. Martins AV, Falcão AL, Silva JE. Estudos sobre os flebótomos do Estado de Minas Gerajs. V. Descrição do macho e da fêmea de Lutzomyia (Lutzomyia) ischyracantha sp.n. (Diptera, Psychodidae). Revista Brasileria de Biologia 22:227-232, 1962.

51. Martins AV, Falcão AL, Silva JE. Nota sobre os flebótomos do Estado de Goiás, com a descrição de duas espécies novas e da fêmea de Lutzomyia longipennis (Barretto, 1946) e a redescrição do macho da $L$. evandroi (Costa Lima \& Antunes, 1936) (Diptera, Psychodidae). Revista Brasileira de Malariologia e Doenças Tropicais 14:379401, 1962.

52. Martins AV, Silva JE. Nova espécie de flebótomo do Estado de Mato Grosso, Lutzomyia (Lutzomyia) dispar sp.n. (Diptera, Psychodidae). Revista Brasileria de Biologia 23:243-246, 1963.

53. Martins AV, Falcão AL, Silva JE. Notas sobre os flebótomos do Território de Roraima, com a descrição de três novas espécies (Diptera, Psychodidae). Revista Brasileira de Biologia 23:333-348, 1963.

54. Martins AV, Silva JE. Notas sobre os flebótomos do Estado do Acre, com a descrição de duas espécies novas (Diptera, Psychodidae). Revista Basileira de Biologia 24:127-138, 1964.

55. Martins AV, Falcão AL, Silva JE. Um novo flebótomo do Estado de Goiás, Lutzomyia teratodes sp.,n. (Diptera, Psychodidae). Revista Brasileira de Biologia 24:321-324, 1964.

56. Martins AV, Falcão AL, Silva JE. Estudos sobre os flebótomos do Estado de Minas Gerais. VI. Descrição de Lutzomyia termitophila sp.n. e Lulzomyia cipoensis sp.n (Diptera, Psychodidae). Revista Brasileira de Biologia 24:309-315, 1964.

57. Martins AV, Falcão AL, Silva JE. Notas sobre os flebótomos do Território de Rondônia, com a descrição de seis espécies novas (Diptera, Psychodidae). Reviosta Brasileira de Biologia 25:1-20, 1965.

58. Martins AV. Esquistossomose mansoni. Relatório apresentado ao $1^{\circ}$ Seminário de Saúde Pública, Fortaleza, CE. Revista da Associação Médica Brasileira 11:397-404, 1965.

59. Martins AV, Silva JE. Estudos sobre os flebótomos do Estado de Minas Gerais. VII. Descrição de Lutzomyia callipygus sp.n. e de Lutzomyia diacantha sp.n. (Diptera, Psychodidae). Revista Brasileira de Biologia25:269-276, 1965.

60. Martins AV, Maciel CS, Silva JE. Notas sobre os flebótomos do grupo squamiventris do subgênero Psychodopygus Mangabeira, 1941. (Diptera, Psychodidae). Boletim do Museu de História Natural da Universidade Federal de Minas Gerais, Série Zoologia 1:1-33, 1968.

61. Martins AV, Silva JE. Barrettomyia n. subg., com descrição de uma espécie nova e da fêmea de Lutzomyia (Barrettomyia) costalimai (Mangabeira, 1942). Boletim do Museu de História Natural da Universidade Federal de Minas Gerais, Série Zoologia, Belo Horizonte 3:1-19, 1968.

62. Martins AV. Epidemiologia da doença de Chagas. In: Cançado JR (ed) Doença de Chagas, Imprensa Oficial, Belo Horizonte p. 225-260, 1968.

63. Martins AV Lutzomyia (Psychodopygus) fairtigi n.sp., from Colômbia (Diptera, Psychodidae, Phlebotominae). Proceedings of the Entomological Society of Washington 72:279, 1970.

64. Martins AV, Coutinho JO, Luz E. Nova espécie de flebótomo do Estado do Paraná: Lutzomyia correalimain.sp. (Diptera, Psychodidae). Revista Brasileira de Malariologia de Doenças Tropicais 22:189-200, 1970.

65. Martins AV, Silva JE, Falcão AL. Estudos sobre os flebótomos do Estado de Minas Gerais. VIII. Descrição de duas novas espécies, Lutzomyia hermanlenti sp.n. e Lutzomyia oliveirai sp.n. (Diptera, Psychodidae, Phlebotominae). Revista 
Brasileita de Biologia 30:547-553, 1970.

66. Martins AV, Silva JE, Falcão AL. Lutzomyia (Lutzomyia) souzalopesi sp.n., nova espécie de feblótomo do Estado do Espírito Santo (Diptera, Psychodidae, Phlebotominae). Revista Brasileira de Biologia 30:559-562, 1970.

67. Martins AV, Lewis DJ, Theodor O. Phlebotomine sandflies. Publication of the World Health Organization, WHO/VBC/71.255, 1971.

68. Masrtins AV, Silva JE, Falcão AL. Nova espécie de flebótomo do Estado do Paraná, Brasil Brumptomyia ortizi sp.n. (Diptera, Psychodidae, Phlebotominae). Boletim do Museu de História Natural da Universidade Federal de Minas Gerais, Série Zoologia 9:1-7, 1971.

69. Martins AV, Fraiha H. Lutzomyia evangelistai sp.n., nova espécie de feblótomo do Estado do Pará (Diptera, Psychodidae, Phlebotominae). Revista Brasileira de Biologia 31:361-365, 1971.

70. Martins AV, Silva JE, Falcão AL. Lutzonyia sherlocki sp.n., do Estado de Mato Grosso, Brasil (Diptera, Psychodidae, phlebotominae). Revista Brasileira de Biologia, 31:415-418, 1971.

71. Martins AV, Falcão AL, Silva JE. Estudos sobre os flebótomos do Estado de Minas Gerais. IX. Descrição de Lutzomyia borgmeieri sp.n. (Diptera, Psychodidae, Phlebotominae). Studia Entomologica, Petrópolis 15:497-502, 1972.

72. Martins AV, Morales-Farias EN. Sobre a distribuição geográfica dos flebotomíneos americanos (Diptera, Psychodidae, Phlebotominae). Revista Brasileira de Biologia 32:361-371, 1972.

73. Martins AV, Falcão AL, Silva JE. Sobre Lutzomyia (Psychodopygus) paraensis (Costa Lima, 1941) e algumas espécies afins. (Diptera, Psychodidae, Phlebotominae). Revista Brasileira de Biologia 33:411-418, 1973.

74. Martins AV, Falcão AL, Silva JE. Notas sobre Lutzomyia (Psychodopygus) davisi (Root, 1934). (Diptera, Psychodidae, Phlebotominae), com a descrição da fềmea. Revista Brasileira de Biologia 33:419-425, 1973.

75. Pessoa SB, Martins AV. Parasitologia Médica. Guanabara Koogan, Rio de Janeiro, 1974.

76. Velasco WJ, Martins AV. Notas sobre Lutzomyia mevesi (Damasceno \& Arouck, 1956), com a descrição da fêmea (Diptera. Psychodidae, Phlebotominae). Revista Brasileira de Biologia 34:93-97, 1974.

77. Martins AV, Falcão AL, Silva JE. Lutzomyia schreiberi n.sp., nova espécie de flebotomíneo a fim de Lutzomyia micropyga (Mangabeira, 1942) (Diptera, Psychodidae, Phlebotominae). Ciência e Cultura 27:765-772, 1975.
78. Martins AV, Falcão AL, Silva JE. Estudos sobre os flebótomos do Estado de Minas Gerais. X. Descrição das fêmeas de Lutzonyia microps (Mangabeira, 1942) e Lutzomyia firmatoi (Barretto, Martins e Pellegrino, 1956)(Diptera, Psychodidae, Phlebotominae). Revista Brasileira de Biologia 35:259-263, 1975.

79. Martins AV, Falcão AL, Silva JE. Estudos sobre os flebótomos do Estado de Minas Gerais. XI. Descrição das fêmeas de Lutzomyia cipoensis Martins, Falcão e Silva, 1964 e de Lutzomyia ischnacantha, Martins, Souza e Falcão, 1962 (Diptera, Psychodidae, Phlebotominae). Revista Brasileira de Biologia 35:297-300, 1975.

80. Martins AV, Falcão AL, Silva JE. Descrição da Fêmea de Luztomyia teratodes Martins, Falcão e Silva, 1964. (Diptera, Psychodidae, Phlebotominae). Revista Brasileira de Biologia 35:515-517, 1975.

81. Martins AV, Llanos BZ, Silva JE. Estudos sobre os flebotomíneos do Perú. (Diptera, Psychodidae, Phlebotominae). 1. Departamento de Cuzco: 1 Descrição de três espécies novas. Revista Brasileira de Biologia 35:645-654, 1975.

82. Llanos BZ, Martins AV, Silva JE. Estudos sobre os flebotomíneos do Perú (Diptera, Psychodidae, Phlebotominae). 1. Departamento de Cuzco: 2 Descrição das fêmeas de Lutzomyia campbelli e Lutzomyia octavioi. Revista Brasileira de Biolo gia 35:655-664, 1975 .

83. Llanos BZ, Martins AV, Silva JE. Estudos sobre os flebotomíneos do Perú (Diptera, Psychodidae, Phlebotominae). 1. Departamento de Cuzco: 3 Descrição do macho e redescrição da fêmea de Lutzomyia (Psychodopygus) amazonensis (Root, 1934) e lista das espécies coletadas. Revista Brasileira de Biologia 35:665-672, 1975.

84. Llanos BZ, Martins AV, Silva JE. Estudos sobre os flebotomíneos do Perú. II. Departamento de Madre de Dios: lista das espécies coletadas e descrição das fêmeas de Lutzomyia micropyga (Mangabeira, 1942) e Lutzomyia calcarala Martins e Silva, 1964 (Diptera, Pschodidae, Phlebotominae). Revista Brasileira de Biologia 36:479-485, 1976.

85. Martins AV, Llanos BZ, Silva JE. Estudos sobre os flebotomíneos do Perú. III. Departamento de Huánuco: Lista das espécies coletadas e descrição de duas espécies novas, Lutzomyia elegans n.sp. e Lutzomyia ruparupa n.sp. (Diptera, Psychodidae, Phlebotominae). Revista Brasileira de Biologia 36:487-494, 1976.

86. Martins AV, Llanos BZ, Silva JE. Estudos sobre os flebotomíneos do Perú. IV. Departamento de Loreto: Lista das espécies coletadas, descrição de 
uma espécie nova, Lutzomyia omagua n.sp. e redescrição do macho de Lutzomyia scaffi (Damasceno \& Arouck, 1956) (Diptera, Psychodidae, Phlebotominae). Revista Brasileira de Biologia 36:495-501, 1976.

87. Ramirez-Perez J, Martins AV, Ramirez A. Lutzomyia conviti, n.sp. de flebotomíneo da Venezuela (Diptera, Psychodidac, Phebotominae). Revista Brasileria de Biologia 36:599-603, 1976.

88. Martins AV, Falcão AL, Silva JE. Estudos sobre os flebótomos do Estado de Minas Gerais. XII. Descrição do macho de Lutzomyia paulwilliamsi n.sp. (Diptera, Psychodidae, Phlebotominae). Revista Brasileira de Biologia 37:135-137, 1977.

89. Martins AV, Falcão AL, Silva JE. Estudos sobre os flebótomos do Estado de Minas Gerais. XIII. Descrição do macho e redescrição da fềmea de Lutzomyia misionensis (Castro, 1960) (Diptera, Psychodidae, Phlebotominae). Revista Brasileria de Biologia 37:569-572, 1977.

90. Martins AV, Falcão AL, Silva JE. Estudos sobre os flebótomos do Estado de Minas Gerais. XIV. Descrição da fềmea de Lutzomyia termitophila Martins, Falcão \& Silva, 1964 (Diptera, Psychodidae, Phlebotominae). Revista Brasileira de Biologia 37:645-647, 1977.

91. Martins AV, Williams P, Falcão AL. American sand flies (Diptera, Psychodidae, Phlebotominae). Monografia publicada na Academia Brasileira de Ciências, Rio de Janeiro, 1978.

92. Martins AV, Falcão AL, Silva JE. Lutzonyia (Nyssomyia) fraihai n.sp., nova espécie de flebotomíneo do Estado da Bahia, Brasil (Diptera, Psychodidae). Revista Brasileira de Biologia 39:357-360, 1979.

93. Martins AV, Ordoñez de Fernandez R, Falcão AL. Flebotomíneos da Venezuela: Lutzonyia torrealbai n.sp., do Estado Trujillo (Diptera, Psychodidae)/. Revista Brasileira de Biologia 39:431-433, 1979.

94. Ramírez-Perez J, Martins AV, Ramírez A. Flebotomíneos da Venezuela: redescrição do macho e descrição da fêmea de Lutzomyia hernandezi (Ortiz, 1965) e descrição da fêmea de Lutzomyia pilosa (Damasceno \& Causey, 1944) (Diptera, Psychodidae, Phlebotominae). Revista Brasileria de Biologia 39:259-264, 1979.

95. Martins AV, Faleão AL, Silva JE, Miranda Filho R. Estudo sobre os flebótomos do Estado de Minas Gerais. XV. Descricão do macho e da fêmea de Latzomyia ranirezi n.sp. e da fêmea de Lutzomyia alencari Martins, Souza e Faleão, 1962. Memórias do Instituto Oswaldo Cruz 77:417-424, 1982.

96. Martins AV, Falcão AL, Silva JE, Miranda Filho
R. Descrição da fêmea de Lutzomyia wilsoni (Damasceno e Causey, 1945) (Diptera, Psychodidae, Phlebotominae). Memórias do Instituto Oswaldo Cruz 78:67-79, 1983.

97. Martins AV, Falcão AL, Silva JE, Miranda Filho R. Nota sobre Lutzomyia (Trichopygomyia) dasypodogeton (Castro, 1939), com a redescrição do macho e da fêmea(Diptera, Psychodidac, Phlebotominae). Memórias do Instituto Oswaldo Cruz 78:223-230, 1983.

98. Martins AV, Falcão AL, Silva JE, Dias ES. Nota sobre Lutzomyia (Lutzomyia) cruzi (Mangabeira, 1938), com a descricão da fêmea (Diptera, Psychodidae, Phlebotominae). Memórias do Instituto Oswaldo Cruz 79:439-442, 1984.

99. Dias ES, Martins AV, Falcão AL, Silva JE. Redescrição do macho e descrição da fêmea de Lutzomyia (Helcocyrtomyia) peresi (Mangabeira, 1942) (Diptera, Psychodidae, Phlebotominae). Memórias do Instituto Oswaldo Cruz 81:215$219,1986$.

100. Dias ES, Martins AV, Falcão AL, Silva JE. Descrição de uma nova espécie do subgênero Helcocyrtomyia, Lutzomyia pusilla sp.n. (Diptera, Psychodidae, Phlebotominae). Memórias do Instituto Oswaldo Cruz 81:395-399, 1986.

101. Dias ES, Falcão AL, Silva JE, Martins AV. Descrição de uma nova espécie do subgênero Helcocyrtomyia, Lutzomhyia capixaba sp.n. (Diptera, Psychodidae, Phlebotominae). Memórias do Instituto Oswaldo Cruz 82:193-196, 1987.

102. Dias ES, Falcão AL, Silva JE, Martins AV. Redescrição do macho e descrição da fêmea de Lutzomyia (Helcocyrtomyia) ferreirana (Barretto, Martins \& Pellegrino, 1958) (Diptera, Psychodidae, Phlebotominae). Memórias do Instituto Oswaldo Cruz 84:245-248, 1989.

103. Martins AV, Dias ES, Falcão AL, Silva JE. Notas sobre os flebótomos dos Estados do Ceará e Piauí, com a descrição da fêmea de Luzomyia samueli (Deane, 1955) (Diptera, Psychodidae, Phlebotominae). Memórias do Instituto Oswaldo Cruz (Enviado e aceito para publicação), 1990.

\section{Prof. Aluizio Prata}

Medicina Tropical /Faculdade de Medicina do Triângulo Mineiro - Uberaba, MG 